Annuaire suisse de politique de développement

$19 \mid 2000$

Pour une politique plus cohérente envers les pays en développement

\title{
8. Aide humanitaire
}

\section{Gérard Perroulaz}

\section{(2) OpenEdition}

12 Journals

Édition électronique

URL : http://journals.openedition.org/aspd/860

DOI : $10.4000 /$ aspd. 860

ISSN : 1663-9669

Éditeur

Institut de hautes études internationales et du développement

Édition imprimée

Date de publication : 1 avril 2000

Pagination : 327-335

ISSN : 1660-5934

\section{Référence électronique}

Gérard Perroulaz, «8. Aide humanitaire », Annuaire suisse de politique de développement [En ligne], 19| 2000, mis en ligne le 19 août 2012, consulté le 08 septembre 2020. URL : http:// journals.openedition.org/aspd/860 ; DOI : https://doi.org/10.4000/aspd.860 


\section{AIDE HUMANITAIRE*}

L 'AIDE HUMANITAIRE de la Confédération s'est élevée à 224,1 millions de francs en 1998. Les principales régions bénéficiaires ont été les Etats de l'ex-Yougoslavie, la région des Grands Lacs (Rwanda), l'Angola et la Corée du Nord. Plusieurs cas d'actions humanitaires entreprises en 1998-1999 montrent les interrelations entre aide humanitaire et coopération au développement à plus long terme. L'aide fournie à l'Amérique centrale suite à l'ouragan «Mitch» s'est greffée sur les relations traditionnelles de coopération que la Suisse entretient avec cette région de concentration. Les cas de la Bosnie et du Kosovo montrent la contribution que la Suisse peut apporter dans le passage de l'aide d'urgence à la reconstruction de cette région, ainsi que les liens entre politique d'aide humanitaire, politique de sécurité (apport du Département fédéral de la défense, de la protection de la population et des sports dans l'action humanitaire, le déminage...) et la politique de la Suisse envers les réfugiés (accueil temporaire de réfugiés de la violence en Suisse et aide au retour). Les sous-chapitres 8.3 et 8.4, qui détaillent l'action de la Suisse en Amérique centrale (suite à l'ouragan «Mitch») et dans les Balkans (Kosovo), évoquent les mesures pour l'action humanitaire et pour une coopération à plus long terme, en soulignant les actions entreprises par plusieurs départements de l'administration fédérale.

L'aide humanitaire de la Confédération est gérée par la Division Aide humanitaire et par le Corps suisse d'aide en cas de catastrophe (ASC), intégrés dans la DDC. Le dernier crédit-cadre sur l'aide humanitaire, d'un montant de 1,05 milliard de francs, a été approuvé par les Chambres fédérales en 1997, pour une durée de quatre ans au moins ${ }^{1}$. Un autre crédit-cadre, renouvelé aussi en 1997, concerne les contributions ordinaires au budget siège du CICR (65 millions de francs par année de 1998 à 2001)². La Suisse est le deuxième pays plus grand financeur du CICR (avec des contributions ordinaires et extraordinaires de près de 82 millions de francs en 1998), derrière les Etats-Unis (127 millions), mais devant l'Union européenne, pour des dépenses annuelles du CICR de 750 à 900 millions de francs.

[Ca ASTM 1998, message sur la continuation de l'aide humanitaire (pp. 324-327).

\subsection{VERSEMENTS D'AIDE HUMANITAIRE EN 1998}

L'aide humanitaire fournie par la Suisse est financée par la Confédération, les cantons et communes suisses, ainsi que les ressources propres des organisations non gouvernementales (ONG). L'aide humanitaire de la Confédération s'est élevée à 224,1 millions de francs en 1998, dont 221,4 millions de francs comptabilisés

* Par Gérard Perroulaz, chargé de recherche à l'IUED.

1. «Message concernant la continuation de l'aide humanitaire internationale de la Confédération» du 20 novembre 1996 (message $\mathrm{n}^{\circ}$ 96.092).

2. «Message concernant l'aide financière de la Confédération au budget siège du Comité international de la CroixRouge (1998-2001)» du 2 juin 1997, Feuille fédérale, 1997, pp. 55-92 (message n 97.047). 
dans l'APD (le reste étant de l'aide publique aux pays de l'Europe centrale et orientale). L'aide humanitaire versée par les cantons et communes s'est élevée la même année à 3 millions de francs (dont 2,1 millions comptabilisés dans l'APD).

La part de l'aide humanitaire représente $17,4 \%$ du total de l'aide publique au développement. Contrairement à ce que l'on pourrait penser, cette part de l'aide humanitaire dans l'aide totale est en diminution ces dernières années. Cela est partiellement dû au fait qu'une grande partie des versements de la Confédération liés à des conflits n'est pour l'instant pas comptabilisée dans l'APD. Certains pays de l'OCDE comptabilisent dans l'aide publique au développement les frais de la première année de l'accueil des réfugiés, alors que la Suisse ne comptabilisait jusqu'en 1999 dans l'APD que l'aide aux réfugiés ou aux personnes déplacées versée sur place ou dans les pays avoisinants. Les cas de la Bosnie et du Kosovo (ce dernier détaillé ci-dessous) montrent aussi qu'une partie de l'aide au retour des réfugiés ainsi qu'une grande partie des activités de promotion de la paix ne sont pour l'instant pas encore comptabilisées dans l'APD.

\section{$\square$ Aide des collectivités publiques locales et des $O N G$}

L'aide humanitaire représente $15 \%$ de l'aide totale versée par les cantons et communes suisses. La part de l'aide humanitaire est par contre beaucoup plus élevée dans l'aide fournie par les ONG, qui est financée par leurs collectes de fonds auprès du public en Suisse. Il faut préciser qu'il est plus facile de récolter de l'argent auprès du public suite à des catastrophes humanitaires que de récolter de l'argent pour un travail de coopération à plus long terme. Les ONG ont versé 59 millions de francs en 1998 pour des actions humanitaires dans les pays en développement et 8,4 millions dans les pays en transition (ce qui représente respectivement $23,6 \%$ et $35 \%$ de l'aide totale fournie par les ONG). Les principales ONG suisses actives dans l'aide humanitaire sont les suivantes: Caritas Suisse (9,6 millions de francs d'aide humanitaire dans les pays en développement en 1998), Kinderhilfe Bethlehem (8,9 millions), Croix-Rouge suisse (6,5), Médecins sans frontières $(5,5)$, Entraide protestante suisse (4).

La Chaîne du bonheur joue un rôle important dans la récolte de fonds auprès du public, souvent suite à des catastrophes naturelles ou des conflits. Ses collectes ont permis de rasssembler près de 56 millions de francs en 1998 (17,9 en 1997), soit un record dans la récolte des fonds. Les principales collectes organisées en 1998 concernaient les régions ou domaines suivants: ouragan «Mitch» en Amérique centrale (34,4 millions de francs récoltés), Sud-Soudan (13,9 millions), Afghanistan (3,5 millions), précarité en Suisse (3,4 millions). En 1999, 20,5 millions de francs ont été versés par la Chaîne du bonheur à diverses œuvres d'entraide pour financer des actions en Suisse ou à l'étranger; cette même année, les récoltes de fonds par la Chaîne du bonheur ont à nouveau atteint un niveau record: plus de 80 millions de francs, dont près de 50 millions de francs récoltés pour le Kosovo.

\section{$\square$ Formes d'intervention de la Confédération}

Le graphique 5 montre la répartition de l'aide humanitaire comprise dans l'APD par types d'intervention, en 1998. Sur les 224 millions de francs d'aide humanitaire, 72,9 millions de francs ont été acheminés par les organisations internationales (dont 30,3 millions par le Programme alimentaire mondial et 28,5 par le 
HCR), 81,9 millions par le CICR et 47,6 millions par le biais des œuvres d'entraide suisses. Les actions directes du Corps suisse d'aide en cas de catastrophe (ASC) se sont élevées à 21,7 millions de francs. L'ASC est intervenu dans 46 pays, avec un engagement particulièrement important dans la région de l'Albanie, de la Bosnie et du Kosovo. Ses autres actions principales ont été menées au Rwanda, au Soudan, en Angola (reconstruction), au Liberia (retour de réfugiés), en Afghanistan (tremblement de terre), en Corée du Nord et en Amérique centrale (ouragan «Mitch»).

L'aide alimentaire s'est élevée à 32,3 millions de francs en 1998. Toute l'aide alimentaire est acheminée par l'intermédiaire des ONG suisses et par le Programme alimentaire mondial (PAM); elle est surtout constituée par du lait en poudre, du maïs, du blé et du riz.

Les quatre domaines d'intervention de l'aide humanitaire comprennent la prévention (surveillance d'activités volcaniques au Guatemala par exemple), le sauvetage (dégagement de blessés), la survie (approvisionnement en eau potable, distribution de nourriture, tentes et abris, équipes d'assistance médicale) et la reconstruction (remise en état des infrastructures et reconstruction de bâtiments publics et habitations).

\section{Graphique 5: Aide humanitaire de la Confédération en 1998}

Répartition selon les formes d'intervention, en millions de francs

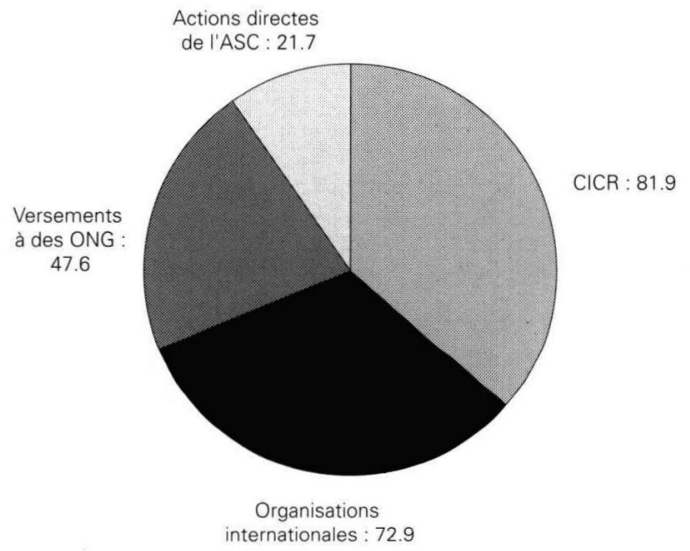

Source: DDC/OFAEE, Coopération internationale de la Suisse. Rapport annuel 1998, brochure "Aide humanitaire", Berne, 1999.

\subsection{PRINCIPALES RÉGIONS BÉNÉFICIAIRES DE L'AIDE HUMANITAIRE}

Le tableau 22 montre les principaux pays ou régions bénéficiaires de l'aide humanitaire en 1998 et l'évolution récente de l'aide envers eux. En prenant compte de toutes les contributions ventilées par pays, les pays du continent africain ont bénéficié de $45 \%$ des versements d'aide de la Confédération, l'Asie en ayant reçu $27 \%$, l'Europe $16 \%$ et l'Amérique latine $12 \%$. En comparaison, l'aide humanitaire des ONG s'est dirigée surtout dans les pays asiatiques (35\% de l'aide humanitaire fournie par les ONG) et africains (33\%), les parts pour 
l'Amérique latine et pour l'Europe étant presque équivalentes à celles attribuées par la Confédération.

Jamais la Suisse n'était intervenue autant que dans l'ex-Yougoslavie. De 1991 à 1997, elle a versé des contributions de plus de 1,5 milliard de dollars (dont 67 millions de dollars proviennent des ONG), en raison des conséquences des conflits en ex-Yougoslavie, dont 1,2 milliard de dollars pour les frais d'accueil des réfugiés en Suisse, 123 millions de dollars pour l'aide humanitaire sur place, 52 millions pour des projets de reconstruction, 35 millions pour les mesures de promotion de la paix, 16 millions pour l'aide au retour. Suite aux accords de Dayton, la Suisse a progressivement passé de l'aide d'urgence à la reconstruction ${ }^{3}$.

Tableau 22: Aide humanitaire de la Confédération (1996 à 1998)

\begin{tabular}{|c|c|c|c|}
\hline \multirow{2}{*}{$\begin{array}{l}\text { Pays ou régions bénéficiaires } \\
\text { de l'aide humanitaire }\end{array}$} & \multicolumn{3}{|c|}{ En millions de francs } \\
\hline & 1996 & 1997 & 1998 \\
\hline 1. Etats de l'ex-Yougoslavie & 16.9 & 13.2 & 13.8 \\
\hline 2. Rwanda/région des Grands Lacs & 18.1 & 9.8 & 8.9 \\
\hline 3. Angola & 9.4 & 8.3 & 7.4 \\
\hline 4. Corée du Nord & 2.8 & 6.3 & 5.6 \\
\hline 5. Afghanistan & 1.8 & 2.7 & 4.3 \\
\hline 6. Soudan & 3.3 & 3.0 & 3.9 \\
\hline 7. Géorgie & 1.0 & 3.9 & 3.8 \\
\hline 8. Amérique centrale & 0.8 & 0.9 & 3.1 \\
\hline 9. Ethiopie & 5.6 & 2.3 & 2.9 \\
\hline 10. Liberia & 2.4 & 1.6 & 2.9 \\
\hline 11. Tadjikistan & 1.8 & 2.1 & 2.8 \\
\hline 12. Sierra Leone & 0.7 & 1.2 & 2.7 \\
\hline 13. Haïti & 1.0 & 0.8 & 2.3 \\
\hline 14. Irak & 2.8 & 2.5 & 2.1 \\
\hline 15. Pérou & 2.2 & 2.0 & 2.0 \\
\hline 15 pays/régions ci-dessus & 70.7 & 60.8 & 68.5 \\
\hline Autres pays & 39.4 & 42.2 & 38.2 \\
\hline Non ventilé géographiquement* & 73.9 & 74.5 & 74.3 \\
\hline Aide humanitaire multilatérale & 46.5 & 44.6 & 43.1 \\
\hline Total de I'aide humanitaire & 230.4 & 222.1 & 224.1 \\
\hline
\end{tabular}

Source: DDC/OFAEE, Coopération internationale de la Suisse. Rapport annuel 1998, brochure "Aide humanitaire", Berne, 1999

* Les actions non ventilées géographiquement comprennent la contribution ordinaire au CICR.

๑ ASTM 1999, dossier consacré à l'action humanitaire (pp. 1-95).

\subsection{OURAGAN «MITCH» EN AMÉRIQUE CENTRALE}

Plusieurs pays d'Amérique centrale ont été sévèrement touchés par l'ouragan «Mitch», qui s'est abattu sur cette région en novembre 1998, provoquant des pertes humaines et des dégâts matériels énormes: plus d'une dizaine de milliers de morts ou de disparus, des millions de sinistrés et de sans-abri, des cultures dévastées et des infrastructures détruites. Au Salvador et au Costa Rica, les

3. Voir la brochure de la DDC Swiss Commitment in Former Yugoslavia, août 1998, qui présente une descrition de l'engagement de la Suisse dans les Etats de l'ex-Yougoslavie. 
deux pays les plus touchés, les dégâts matériels ont été évalués à 3,2 milliards de dollars ${ }^{4}$.

Beaucoup d'ONG, dont Caritas Suisse, la Croix-Rouge suisse, l'Entraide protestante suisse (EPER) et l'Euvre suisse d'entraide ouvrière (OSEO), se sont mobilisées suite à l'ouragan pour récolter des fonds en Suisse et apporter une aide humanitaire. La Chaîne du bonheur a même enregistré un montant record de dons lors de sa campagne de récolte de fonds, qui ont atteint 34,4 millions de francs. En mai 1999, à Stockholm, la Suisse a participé à la Conférence des donateurs pour la reconstruction et la transformation de l'Amérique centrale, au cours de laquelle les pays d'Amérique centrale et des $\mathrm{ONG}^{5}$ ont réclamé un allègement de la dette, afin de leur permettre de faire face à une partie des coûts énormes de reconstruction. Plusieurs ONG (dont des ONG suisses) se sont plaintes de la réticence du Nicaragua à coopérer avec elles pour acheminer l'aide aux victimes ${ }^{6}$.

Les moyens que la Confédération suisse met à la disposition des pays victimes de l'ouragan «Mitch» s'élèvent à 43 millions de francs d'ici la fin de l'an 2000 (23 millions versés par le Seco et 20 millions par la DDC). Le Corps suisse d'aide en cas de catastrophe a été mobilisé, ainsi que les collaborateurs permanents de la DDC. Les projets de la DDC concernent surtout le Honduras et le Nicaragua, dans les domaines de la reconstruction des infrastructures sociales (écoles et hôpitaux) et de la réparation des installations d'approvisionnement en eau potable. La Suisse a réorienté l'aide au développement accordée depuis de nombreuses années aux pays d'Amérique centrale vers les victimes de «Mitch».

Le Seco a accordé une aide de 13 millions de francs en faveur du Nicaragua, dont 5 millions d'aide extraordinaire à la balance des paiements pour financer l'importation de pièces de rechange pour la réparation des dommages causés dans des usines électriques et 8 millions pour l'amortissement de dettes multilatérales; il a également versé 10 millions au Honduras pour l'amortissement de dettes multilatérales (couverture d'une partie du service de la dette du Honduras, service de la dette qui représente $40 \%$ des revenus de l'Etat $)^{7}$. Ce dernier paiement s'est effectué par l'intermédiaire du compte fiduciaire spécial de la Banque mondiale prévu pour l'Amérique centrale. Pour le Seco, les demandes d'allègement de la dette allaient dans le sens des mesures déjà prises par la Suisse dans le cadre de son programme de désendettement. En effet, la Suisse avait déjà remis l'ensemble de la dette que ces pays avaient envers elle, avec la création des fonds de contrepartie $^{8}$. Le solde du Fonds de contrepartie au Honduras issu de l'accord bilatéral de désendettement conclu en 1993 a été réaffecté à des projets d'aide à la reconstruction. Pour Nicolas Imboden (Seco), il n'est pas pour autant question

4. Selon une estimation du Programme alimentaire mondial.

5. Notamment la coalition d'ONG demandant des remises de dettes, Jubilée 2000

6. "Aide suisse aux victimes de "Mitch"», Le Courrier, 10.11.98.

7. DFAE/DFE, Communiqué de presse, 28.5.99; OFAEE, Communiqué de presse, 12.11.98; Seco, Communiqué de presse, 12.8.99; «Humanitäre Soforthilfe für Zentralamerika», Neue Zürcher Zeitung, 11.11.98.

8. Au Honduras, la Suisse a annulé en 1993 l'ensemble de la dette bilatérale de 63,3 millions de francs et a contribué, à raison de 14 millions de francs, à l'acquittement de la dette de ce pays envers les institutions financières multilatérales. Au Nicaragua, la Suisse a contribué en 1991 au paiement des arriérés envers les institutions financières multilatérales, arriérés consécutifs à la suspension, par les sandinistes, du service de la dette. En 1993, la Suisse a annulé la dette bilatérale de 2,6 millions de francs et, en 1995, a contribué pour 12 millions de francs au rachat d'une dette commerciale. Avec d'autres donateurs, elle s'est aussi engagée à couvrir le service de la dette multilatérale dû jusqu'à fin 1998. Source: OFAEE, Communiqué de presse, 12.11.98. 
que la dette soit annulée unilatéralement, car les gouvernements doivent respecter les programmes d'ajustement structurel qui leur assurent une position tenable en matière de finances extérieures ${ }^{9}$.

\subsection{AIDE AU KOSOVO}

L'aide humanitaire suisse en Albanie, en Macédoine et en République fédérale de Yougoslavie (Serbie, Monténégro et Kosovo) s'est organisée par le biais de l'aide bilatérale (Division Aide humanitaire et Corps suisse d'aide en cas de catastrophe), de l'aide multilatérale (Programme alimentaire mondial, initiative Focus, participation aux forces multinationales KFOR), ainsi que de l'aide fournie par d'autres départements de l'administration fédérale (programme d'aide au retour des réfugiés accueillis temporairement en Suisse, versée par l'ODR, et engagement du DDPS). L'aide passe par une très large palette de mesures, souvent nouvelles et originales, telles l'initiative Focus et l'aide pour ceux qui accueillent des réfugiés kosovars en Albanie, ou reprises de l'expérience bosniaque (aide au retour des réfugiés ${ }^{10}$.

Le Conseil fédéral a accordé un crédit de 110 millions de francs à l'aide humanitaire dans le cadre du conflit au Kosovo, dont 80 millions de francs ont été utilisés jusqu’à fin novembre 1999) ${ }^{11}$.

\section{$\square$ Aide humanitaire (DDC, DFAE)}

L'aide humanitaire suisse a mis l'accent sur l'aide aux réfugiés dans les pays avoisinants (surtout l'Albanie et la Macédoine), puis sur le soutien aux personnes qui sont ensuite retournées au Kosovo. Le Corps suisse d'aide en cas de catastrophes (ASC) a d'abord participé à l'aide d'urgence dans les camps de réfugiés. Suite à la fin des bombardements et le retrait des forces serbes, l'aide de la DDC et de l'ASC s'est concentrée sur la reconstruction et l'aide à plus long terme. Voici des exemples illustrant les principales actions humanitaires coordonnées par la DDC:

」 pour favoriser l'aide aux réfugiés kosovars qui restent dans la région, la DDC a instauré, avec l'aide d'ONG et du HCR, un système d'aide financière aux familles albanaises qui accueillent des réfugiés du Kosovo, le Cash for Shelter («de l'argent pour des abris»), se montant à 10 millions de francs;

- l'opération, menée par la DDC, l'Office fédéral de l'agriculture et l'Union suisse des paysans, Des vaches pour le Kosovo, soit l'envoi de 500 vaches suisses, de tracteurs et de semences, permettra entre autres de reconstituer un cheptel décimé par la guerre et d'approvisionner la population en lait;

9. «Le cyclone "Mitch" a rendu explosive la dette de l'Amérique centrale», Le Temps, 10.11.98; «Remise de dettes, envoi de militaires: l'aide internationale prend corps en Amérique centrale», Le Temps, 11.11.98.

10. Les mesures d'aide suisse au Kosovo ont été abondamment décrites dans la presse et dans certaines publications de la DDC (Service de presse, Communiqués de presse, Newsletter). Des informations hebdomadaires sont données sur le site Internet du Bulletin des Balkans, publication conjointe du DFAE, du DFJP et du DDPS: www.eda.admin.ch/ site/f/publikationen/balkanbulletin.html.

11. A cette somme de 110 millions, prélevée sur le crédit-cadre d'aide humanitaire bilatérale et multilatérale (et donc incluse dans l'aide publique au développement), s'ajoutent les versements de 55 millions de francs du DDPS pour l'envoi des militaires de la Swisscoy, 10 millions pour le déminage et les missions de l'OSCE et 33 millions dans le cadre du Pacte de stabilité pour l'Europe du Sud-Est (aide aux pays qui ont souffert de la guerre: Albanie, Macédoine, Bulgarie, Hongrie, Roumanie), ainsi qu'une somme estimée à 250 millions de francs pour l'aide au retour. La Suisse accorde ainsi en tout plus de 200 millions de francs en 1999-2000 suite au conflit au Kosovo, sans compter l'accueil des réfugiés en Suisse. Pour comparaison, l'intervention du Corps suisse d'aide en cas de catastrophe dans 47 pays a représenté une dépense de 21,7 millions de francs en 1998. 
- reconstruction: l'aide à la reconstruction comprend la remise en état et la construction d'immeubles locatifs pour loger les réfugiés qui retournent au pays, la rénovation d'écoles et de centres de santé, la réhabilitation des réseaux d'eau potable;

- l'initiative Focus, organisée dès fin mars 1999 conjointement par la Suisse, l'Autriche, la Grèce et la Russie, a permis d'acheminer rapidement une aide d'urgence en République fédérale de Yougoslavie, en attendant que d'autres organisations humanitaires soient actives sur ce territoire ${ }^{12}$. L'initiative s'est ensuite poursuivie, notamment pour fournir une aide aux populations les plus vulnérables à l'approche de l'hiver. 1100 tonnes de matériel humanitaire sont ainsi parvenues au Kosovo d'avril à septembre 1999 (nourriture, couvertures, sacs de couchage, matelas et tentes). D'autres projets concernent le rétablissement de systèmes de chauffage, des opérations de déminage et la réhabilitation de plusieurs écoles et habitations dans la province du Kosovo, ainsi que la décontamination de sites pollués par des produits toxiques. La neutralisation et le stockage de mercure échappé du site industriel de Pancevo constituent un des projets de décontamination, afin de remédier aux conséquences d'une catastrophe écologique provoquée par les bombardements de l'OTAN qui ont détruit ce site industriel.

Fin novembre 1999, la DDC annonçait une aide alimentaire en trois volets pour un montant de 5,6 millions de francs, destinée au Kosovo et à la Serbie, prélevée sur le crédit global de 110 millions et comprise dans l'agrégat d'APD:

- les paysans suisses acceptant de vendre du froment à la Fédération suisse des producteurs de céréales à un prix moins élevé, celle-ci est en mesure d'offrir à la DDC 730 tonnes de farine, de sorte que la DDC n'a plus que les frais de transport, soit 1 demi-million de francs, à sa charge (cette farine permet de faire du pain pour 60'000 personnes pendant un mois);

- l'ASC fournit 500 tonnes d'huile de colza pour une valeur de 1,6 million de francs (huile à prix bas car subventionnée par l'Office fédéral de l'agriculture à hauteur de 1 demi-million de francs);

- le troisième volet de l'aide comprend une contribution au Programme alimentaire mondial (PAM) pour l'achat et la distribution de 5280 tonnes de farine de blé (montant de 3,5 millions de francs qui s'ajoute à la contribution annuelle ordinaire de 21 millions de francs que la Suisse verse au PAM) ${ }^{13}$.

Un nombre très important d'ONG européennes, dont plusieurs ONG suisses, se sont associées à l'aide humanitaire, avec par exemple les projets de l'OSEO ${ }^{14}$ et de Caritas Suisse pour la reconstruction d'habitations (la Croix-Rouge suisse et l'EPER étant les deux autres principales ONG actives dans la région). La DDC a versé au total plus de 80 millions de francs en 1999 à l'aide humanitaire au Kosovo, dont 35 millions pour les organisations internationales, essentiellement le HCR, le CICR et le PAM.

12. L'initiative Focus a été critiquée par certains comme une étrange alliance avec les autres pays partenaires (dont quelques-uns sont plutôt pro-serbes), et qui fait double emploi avec le travail du CICR ou d'autres organisations. Pour la DDC, il était important d'organiser l'acheminement de l'aide d'urgence alors que le Kosovo était encore sous occupation des forces armées serbes et sous les bombardements de l'OTAN, et que d'autres organisations n'y étaient pas encore présentes (Le Courrier, 17.5.99).

13. DDC, «Farine de blé et huile de colza suisses pour le Kosovo et la Serbie», Communiqué de presse, 29.11.999.

14. «Schweizer Hilfe für den Wiederaufbau von Häusern in Kosovo», Neue Zürcher Zeitung, 13-14.11.99. 


\section{$\square$ Coopération technique (DDC, DFAE)}

Depuis le $1^{\text {er }}$ juillet 1999, la Suisse finance le studio de radio de l'UNMIK (administration transitoire des Nations Unies au Kosovo). Cette radio indépendante, dirigée par la Fondation Hirondelle, diffuse des informations en albanais et en serbe $^{15}$. L'objectif est la diffusion d'informations rigoureuses avec un large éventail d'opinions pour contribuer au pluralisme et au débat démocratique. Les dépenses liées à la coopération technique se sont élevées à environ 4 millions de francs en 1999. La DDC prépare un programme de coopération technique plus important pour l'année 2000 (approvisionnement en eau potable, assainissement, agriculture, santé et éducation).

D'autres mesures complètent l'aide humanitaire et la coopération technique fournie par la DDC. Ces mesures, qui pour l'instant ne sont pas ou seulement partiellement comprises dans l'agrégat d'aide publique au développement, ont été menées par l'Office fédéral des réfugiés ou le DDPS.

\section{$\square$ Programme d'aide au retour (Office fédéral des réfugiés, DFJP)}

Près de 12 '000 personnes ont quitté la Suisse de juillet à décembre 1999 pour revenir au Kosovo, sur 50’000 à 60'000 Kosovars présents en Suisse. Un programme d'aide au retour, qui s'inspire du cas bosniaque, a été instauré pour inciter les réfugiés à rentrer au pays en offrant des primes individuelles aux «volontaires» pour ce retour; celui-ci s'effectue en plusieurs phases, dont la période de retour volontaire (jusqu'au 31 décembre 1999) avec une assistance au retour plus élevée, puis la période de départ obligatoire (jusqu'au 31 mai 2000).

L'aide au retour individuelle pour les réfugiés venus en Suisse est versée par l'Office fédéral des réfugiés (et non comprise dans 1'APD). Chaque réfugié qui retourne au Kosovo bénéficiait en 1999 d'une aide individuelle à la réinstallation de 2000 francs par adulte et de1000 francs par mineur, un montant complémentaire de 3000 francs par famille étant de plus engagé dans l'aide matérielle sur place (matériel pour la réparation ou la reconstruction de leurs habitations). Ces montants ont été réduits de $50 \%$ pour les réfugiés qui retournent au Kosovo dès le $1^{\text {er }}$ janvier 2000 . Les candidats au retour peuvent dorénavant choisir entre une aide matérielle sur place ou une contribution financière aux frais de déménagement. Le coût total du programme d'aide au retour est évalué à 250 millions de francs sur une période de trois ou quatre ans (5000 francs en moyenne par réfugié d'aide individuelle au retour et d'aide à la reconstruction ou à la rénovation de logement).

ASTM 2000, sous-chapitre 5.3, partie «Accueil des réfugiés kosovars: politique de renvoi et aide au retour».

- Actions menées par le Département fédéral de la défense, de la protection de la population et des sports (DDPS)

๖ Envois d'hélicoptères de l'armée suisse (Super Puma) en Albanie pour l'acheminement de l'aide d'urgence.

- Participation de la Suisse à l'engagement des forces multinationales de paix au Kosovo (KFOR), par le détachement suisse non armé nommé Swisscoy, avec des personnes recrutées et payées par le DDPS, qui dispose d'un budget

15. Voir le site Internet de la Fondation Hirondelle www.hirondelle.org. 
de 55 millions de francs pour cet engagement, sur des fonds non compris dans l'APD $^{16}$. Le détachement participe notamment à des travaux de reconstruction d'immeubles et de bâtiments scolaires, ainsi que de remise en état du réseau électrique et de l'alimentation en eau.

- Envois d'experts suisses pour la mission de l'OSCE, de policiers pour la force internationale de police de l'ONU, et de médecins légistes pour les enquêtes sur les violations des droits de l'homme (enquêtes menées par le Tribunal pénal international).

ASTM 2000, sous-chapitre 5.1.4, partie «Engagements militaires hors de Suisse».

\section{SOURCES}

ASC, SKH-Journal, Berne, trimestriel.

DDC (DFAE), Communiqués de presse, aide humanitaire pour le Kosovo, 28.4.99; «La Suisse prend une part active à la reconstruction au Kosovo», 13.7.99; initiative Focus, 30.7.99; initiative Focus, 6.10.99; «Farine de blé et huile de colza suisses pour le Kosovo et la Serbie», 29.11.99.

DDPS/DFAE/DFJP, Bulletin des Balkans. Informations hebdomadaires, www.eda.admin.ch/site/f/publikationen/balkanbulletin.html.

Division Aide humanitaire/ASC (DDC), L'aide humanitaire internationale de la Confédération (information de base), janvier 1998.

M. Mercier, De Sarajevo à Pristina. L'engagement de la Chaîne du bonheur dans les Balkans de 1992 à 1999, Chaîne du bonheur, 2000 (disponible également sur le site Internet de la Chaîne du bonheur www.bonheur.ch).

La Liberté, initiative Focus, 11.5.99; aide au Kosovo, 24.6.99.

Neue Zürcher Zeitung, «Hilfe für Kosovo-Flüchtlinge an Ort und Stelle», 24.3.99; «20 Millionen für Opfer des Kosovokonflikts», 1.4.99; «Ausweitung der Soforthilfe für Kosovo», 6.4.99; «Humanitäre Hilfe der Schweiz für Kosovo», 7.4.99; «Bargeld gegen Behausung», 15.4.99; «Schweizer Beteiligung an Kosovo-Friedenstruppe», 24.6.99; «Schweizer Hilfe für den Wiederaufbau von Häusern in Kosovo», 13-14.11.99.

SITES INTERNET ET COMMANDE DE PUBLICATIONS

ASC: www.skh.ch.

Chaîne du bonheur: www.bonheur.ch.

DDC: www.sdc-gov.ch.

Commande des publications de la DDC au service de l'information, Freiburgstrasse 130, 3003 Berne. 